\title{
Utilization of HPASubC for the identification of sinusoid-specific proteins in the liver
}

Divine-Favour Anene ${ }^{1}$, Avi Z. Rosenberg ${ }^{2}$, David E. Kleiner ${ }^{3}$, Toby C. Cornish ${ }^{1,4}$, Marc K. Halushka ${ }^{1}$

1. Department of Pathology, Johns Hopkins University School of Medicine, 720 Rutland Avenue, Baltimore, MD, 21205 United States

2. Department of Pathology, Children's National Medical Center, 111 Michigan Ave, NW Washington, DC, 20010, United States

3. Laboratory of Pathology/National Cancer Institute, Bldg 10, Room 2S235, MSC 1500, 10 Center Dr. Bethesda, MD, 20892, United States

4. Department of Pathology, University of Colorado School of Medicine, Academic Office 1, Rm L152109, 12631 East 17th Avenue, Aurora, CO 80045, United States

\section{Supporting Information}

Figure S-1

Table S-1 List of putative Kupffer cell proteins

Table S-2 List of putative Endothelial cell proteins

Table S-3 List of putative Lymphocyte proteins

Table S-4 List of putative Stellate cell proteins

Table S-5 List of putative Sinusoidal (undistinguished) proteins

Table S- 6 List of putative proteins identified by HPASubC but not present in two mass-spectrometry based liver proteomic datasets

Table S-7: List of generic proteins
Page S-2

Page S-3

Page S-3

Page S-3

Page S-3

Page S-3
Page S-3

Page S-3 
Figure S-1
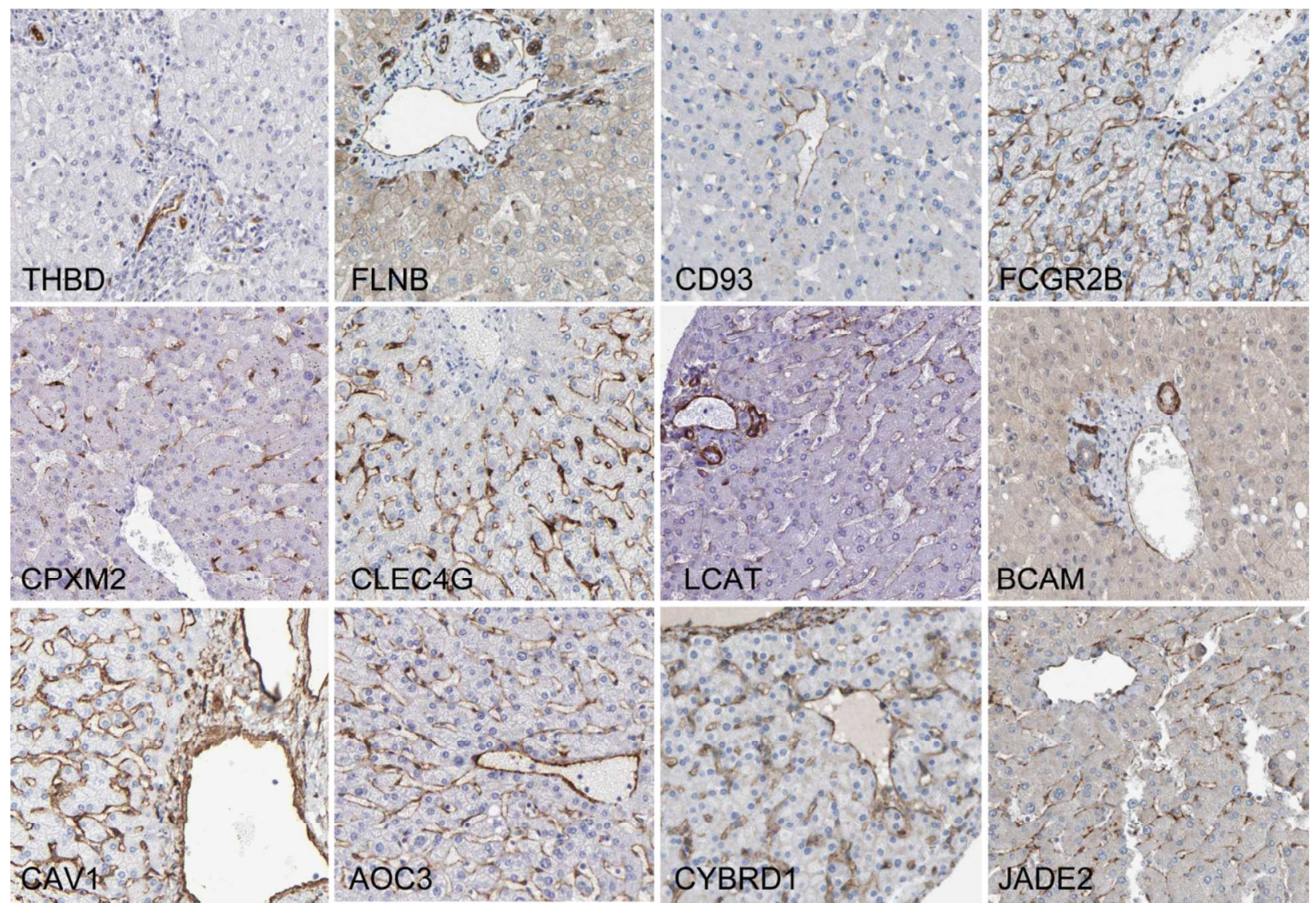

Figure S-1. Additional patterns of endothelial cell staining in the liver. These proteins were reported in Figure 4. Images from Human Protein Atlas. 
Table S-1 List of putative Kupffer cell proteins

Table S-2 List of putative Endothelial cell proteins

Table S-3 List of putative Lymphocyte proteins

Table S-4 List of putative Stellate cell proteins

Table S-5 List of putative Sinusoidal (undistinguished) proteins

Table S- 6 List of putative proteins identified by HPASubC but not present in two mass-spectrometry based liver proteomic datasets. In the FPKM column, “--" indicates no available FPKM data.

Table S-7: List of generic proteins

(Provided in Excel Format File) 